University of Wollongong

Research Online

Faculty of Engineering - Papers (Archive)

Faculty of Engineering and Information

Sciences

April 2004

\title{
Optimum dynamic balancing of planar parallel manipulators
}

G. Alici

University of Wollongong, gursel@uow.edu.au

B. Shirinzadeh

Monash University

Follow this and additional works at: https://ro.uow.edu.au/engpapers

Part of the Engineering Commons

https://ro.uow.edu.au/engpapers/25

\section{Recommended Citation}

Alici, G. and Shirinzadeh, B.: Optimum dynamic balancing of planar parallel manipulators 2004.

https://ro.uow.edu.au/engpapers/25

Research Online is the open access institutional repository for the University of Wollongong. For further information contact the UOW Library: research-pubs@uow.edu.au 


\section{Optimum Dynamic Balancing of Planar Parallel Manipulators}

\author{
Gürsel Alıcı \\ School of Mechanical, Materials \\ and Mechatronics Engineering \\ University of Wollongong \\ 2522, NSW, Australia.
}

\author{
Bijan Shirinzadeh \\ Robotics \& Mechatronics Research Laboratory \\ Department of Mechanical Engineering \\ Monash University \\ 3800 , VIC, Australia
}

\begin{abstract}
This paper presents a methodology for optimum dynamic balancing of planar parallel manipulators typified with a variable speed 2 DOF parallel manipulator articulated with revolute joints. The dynamic balancing is formulated as an optimisation problem such that a sum-squared values of bearing forces, driving torques, shaking moment, and the deviation of the angular momentum from its mean value are minimized throughout an operation range of the manipulator, provided that a set of balancing constraints consisting of the shaking force balancing conditions, the sizes of some inertial and geometric parameters are satisfied. Sets of optimisation results corresponding to various combinations of the elements of the objective function are evaluated in order to quantify their influence on the resulting bearing forces, the driving torques, shaking moment and force. The results prove that the proposed optimisation approach can be used to minimize any desired combination of the forces, moments, and torques involved in any parallel mechanism by choosing a suitable set of weighting factors. The method is systematic, versatile and easy to implement for the optimum balancing of the parallel manipulator and more general parallel manipulators.
\end{abstract}

Index Terms - dynamic balancing, parallel manipulators, optimum mechanism design.

\section{INTRODUCTION}

Parallel manipulators or in-parallel actuated mechanisms, which consist of one or more closed kinematic chains, have the advantages of high stiffness, good dynamic characteristics, and precise positioning capability [1]. A revolute-jointed five-bar mechanism is the only example of the multi input mechanisms having practical importance, especially for following any arbitrary planar curve precisely which can not be realized with single degree of freedom mechanisms such as four-bar and slider crank mechanisms [1-5]. It has, therefore, been recognised as the most simple and useful planar parallel robot manipulator [5].

When designing high-speed mechanisms such as a parallel manipulator, a special attention should be paid to the inertiainduced force (shaking force) and moment (shaking moment) transmitted to the mechanism frame. If their magnitudes and directions change throughout the operation of the mechanism, the mechanism will vibrate undesirably, and consequently, its dynamic performance will be unsatisfactory. Therefore, the designer's main concern is either to completely eliminate them or to ensure that their magnitudes and directions do not change significantly. If the former is realized, such a mechanism is said to be dynamically balanced. It is virtually impossible to have the former realized without increasing the number of moving links in the mechanism. The latter can be realized by taking certain precautions such as minimising the magnitudes of the inertia-induced force and moment, and if possible constant. It is the aim of this paper to follow the latter approach such that the mechanism is dynamically balanced with minimum bearing forces as well as it requires minimum driving torques. Based on a constrained optimization procedure, this is accomplished by selecting a proper set of the mass distribution parameters of the moving links. Of course, distributing or redistributing (in the form of adding counterweights) the mass of the links may increase the inertia forces as well as bearing, and ground forces, and driving torques needed to move the mechanism. We, therefore, employ a non-linear programming method in the selection of the balancing parameters of the moving links to ensure that the manipulator is optimum with respect to all the bearing forces, the driving torques, and shaking force and moment. The optimisation results prove that the method is systematic, versatile and easy to implement for the optimum dynamic balancing of parallel manipulators.

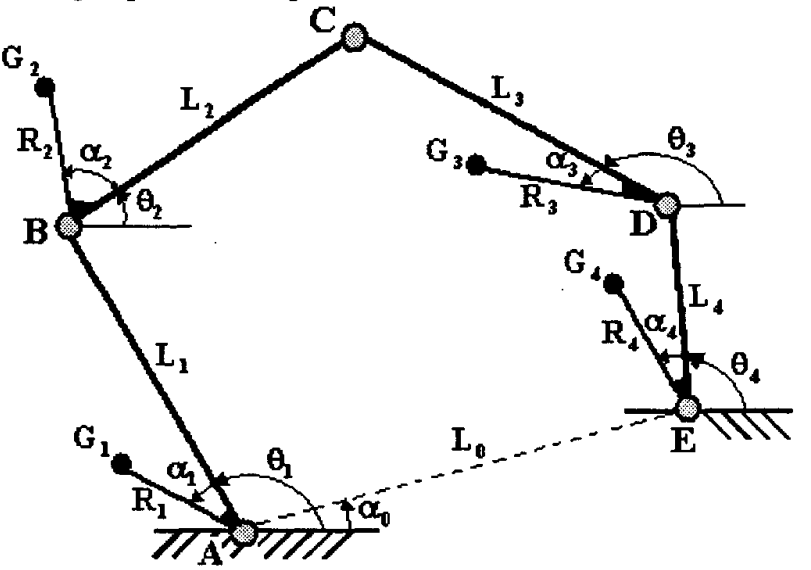

Figure 1. Schematic of the planar manipulator with mass centre location parameters.

There is a wealth of literature on the dynamic balancing of single degree of freedom mechanisms [7-10], static balancing of planar and spatial parallel manipulators [11,17], and optimisation procedures based on the minimization of the force 
and moment transmitted to the ground [12-14]. However, very little has been published on the optimum dynamic balancing of planar parallel manipulators. Wiederrich and Roth [14] presented general conditions to determine the mass distribution parameters of four-bar mechanisms operating in the horizontal plane, based on the linear momentum and angular momentum balancing. It was assumed that the mass of the mechanism could be represented with a point mass, without any mass moment inertia about the mass center. Conte, George, Mayne, and Sadler [19] reported on a balancing method that combined kinematic synthesis, dynamic design, and input speed trajectory design to reach the trade-off of dynamic balance and to satisfy the kinematic requirements and constraints simultaneously for four-bar linkages. Feng [20] have used a combination of mass distribution and the addition of two types of the inertia counterweights in order to realize the dynamic balancing of a number of single degree freedom mechanisms. Arakelian and Dahan [21] studied the shaking force and shaking moment balancing of a four-bar mechanism and a spatial RSSR mechanism by minimizing the RMS value of the shaking moment. A considerable amount of research has also been devoted to balancing methodologies based on shaking force and moment transmitted to the frame of the mechanisms, predominantly single degree of freedom mechanisms, as provided in $[8,15]$.

\section{FIVE-BAR MANIPULATOR WITH REVOLUTE JOINTS}

The five-bar planar manipulator considered in this study is shown in Fig. 1, where its two joints (A and E) connected to the ground are active and the others are passive joints. The input motions of the active joints can be independent from each other or be provided via a set of gears maintaining a specified phase angle between the two active joints [6]. Analytical expressions for the coordinates of the output point $C$, where the end effector is connected, are obtained for the provided joint inputs $\theta_{1}$ and $\theta_{4}$, and the specified link lengths $L_{0}, L_{1}, L_{2}, L_{3}, L_{4}$ and the angle $\alpha_{0}$ [16]. It must be noted that for a parallel RRRRR manipulator, $L_{1}=L_{4}$ and $L_{2}=L_{3}$. The angle $\alpha_{i}$ and the radial distance $R_{i}$ describe the mass center $G_{i}$ of the $i^{\text {ih }}$ link.

\section{FORCE ANALYSIS}

The aim of the force analysis is to obtain analytical expressions for the forces acting on the bearings A, B, C, D, and $\mathrm{E}$, and driving torques required to move the mechanism with a variable speed. For the sake of brevity, the free-body diagrams of the links are omitted here. It is assumed that there is no friction in the system. The forces $F_{A x}, F_{A y}, F_{B x}, F_{B y}$, $F_{C x}, F_{C y}, F_{D x}, F_{D y}, F_{E x}, F_{E y}$ acting at the joints and the driving torques $\tau_{1}$ and $\tau_{2}$ are obtained from the dynamic force and moment equations written for each link of the manipulator. The resulting equations may be represented in a matrix-vector form;

$$
[M][F]=\left\lfloor F_{s}\right\rfloor
$$

where $\mathrm{M}, \mathrm{F}$, and $F_{g}$ denote the square matrix of known mechanism dimensional parameters and joint angles, the vector of unknown forces and torques, and the vector of gravitational and inertial forces, and inertial torques, respectively.

\section{FORMULATION OF OPTIMUM BALANCING}

A dynamically balanced mechanism requires that the shaking force and the shaking moment, which are due to moving inertia of the system, transmitted to the frame of the mechanism are zero. This is an ideal requirement that cannot be satisfied with the mass redistribution only. This follows that, as it has widely been reported in the literature $[8,20,21]$, the complete shaking force and shaking moment is possible with the addition of some auxiliary linkages. This of course will increase the number of links and increase the burden on the actuators and bearing forces. Another possible solution is to minimize their magnitudes about their average values.

\section{A. Shaking Force Balancing Requirements}

The shaking force can be expressed as the time rate of change of the linear momentum of the system with respect to a point in the mechanism frame [23]. This follows that if the linear momentum of the mechanism is constant throughout its operation range, the shaking force transmitted to the frame is zero. The linear momentum $L_{A}$ of a system consisting of ' $n$ ' interconnected rigid bodies is;

$$
\vec{L}_{A}=\sum_{i=\mathrm{t}}^{n-1} m_{i} \overrightarrow{\dot{r}}_{i}
$$

The shaking force transmitted to the frame is then

$$
\vec{F}_{s}=-\frac{d \vec{L}_{A}}{d t}
$$

Using the notation given in Fig. 1, the position vector $\vec{R}$ for the mass center of the mechanism is

$$
\vec{R}=\frac{1}{M_{T}} \sum_{i=1}^{n-1} m_{i} \vec{r}_{i}
$$

where $\vec{r}_{i}$ is the position vector for the mass center $G_{i}$ of the $i^{\text {th }}$ moving link with a mass of $m_{i}$ with respect to the reference point $A$. With this in mind, the linear momentum can also be expressed as

$$
\vec{L}_{A}=M_{T} \overrightarrow{\dot{R}}
$$

where $M_{T}$ is the total mass of the moving links. The individual position vectors $\vec{r}_{i}$ are expressed in complex numbers as 


$$
\begin{aligned}
& \vec{r}_{1}=R_{1} e^{j\left(\theta_{1}+\alpha_{1}\right)}, \quad \vec{r}_{2}=L_{1} e^{j \theta_{1}}+R_{2} e^{j\left(\theta_{2}+a_{2}\right)}, \\
& \vec{r}_{3}=L_{0} e^{j \alpha_{0}}+L_{4} e^{j \theta_{4}}+R_{3} e^{j\left(\theta_{3}+a_{3}\right)}, \\
& \vec{r}_{4}=L_{0} e^{j a_{0}}+R_{4} e^{j\left(\theta_{4}+\alpha_{4}\right)}
\end{aligned}
$$

The exponential terms are related to each other by the loop closure equation

$$
L_{1} e^{j \theta_{1}}+L_{2} e^{j \theta_{2}}=L_{0} e^{j \alpha_{0}}+L_{3} e^{j \theta_{3}}+L_{4} e^{j \theta_{4}}
$$

The exponential term $e^{j \theta_{3}}$ (or $e^{j \theta_{2}}$ ) is extracted from (7), and then substituted into (6). Now, the position vectors in (6) are substituted into (4) from which the position vector describing the overall mass center of the mechanism is obtained as

$$
\vec{R}=\frac{1}{M_{T}}\left[\begin{array}{l}
e^{\beta_{1}}\left(m_{1} R_{1} e^{j \alpha_{1}}+\frac{m_{3} R_{3} L_{1}}{L_{3}} e^{j \alpha_{3}}+m_{2} L_{1}\right) \\
+e^{j 0_{2}}\left(m_{2} R_{2} e^{j \alpha_{2}}+\frac{m_{3} R_{3} L_{2}}{L_{3}} e^{j \alpha_{3}}\right) \\
+e^{j 0_{4}}\left(m_{4} R_{4} e^{j \alpha_{4}}-\frac{m_{3} R_{3} L_{4}}{L_{3}} e^{j \alpha_{3}}+m_{3} L_{4}\right) \\
+L_{0} e^{j \alpha_{0}}\left(-\frac{m_{3} R_{3}}{L_{3}} e^{j \alpha_{3}}+m_{3}+m_{4}\right)
\end{array}\right]
$$

After taking the first time derivative of (8) and substituting into (5), the analytical expression for the linear momentum of the system is obtained as;

$$
\vec{L}_{A}=\left[\begin{array}{l}
\left(j \dot{\theta}_{1}\right) e^{j 0_{1}}\left(m_{1} R_{1} e^{j \alpha_{1}}+\frac{m_{3} R_{3} L_{1}}{L_{3}} e^{j \alpha_{3}}+m_{2} L_{4}\right) \\
+\left(j \dot{\theta}_{2}\right) e^{j \theta_{2}}\left(m_{2} R_{2} e^{j \alpha_{2}}+\frac{m_{3} R_{3} L_{2}}{L_{3}} e^{j \alpha_{3}}\right) \\
+\left(j \dot{\theta}_{4}\right) e^{j \theta_{4}}\left(m_{4} R_{4} e^{j \alpha_{4}}-\frac{m_{3} R_{3} L_{4}}{L_{3}} e^{j \alpha_{3}}+m_{3} L_{4}\right)
\end{array}\right]
$$

If the coefficients of $\left(j \dot{\theta}_{k}\right) e^{j \theta_{k}}$ become zero, the linear momentum of the system will be zero. To this end, the following shaking force balancing conditions are found;

$$
\begin{gathered}
\left(m_{1} R_{1} \cos \alpha_{1}+\frac{m_{3} R_{3} L_{1}}{L_{3}} \cos \alpha_{3}+m_{2} L_{1}\right)=0 \\
\left(m_{1} R_{1} \sin \alpha_{1}+\frac{m_{3} R_{3} L_{1}}{L_{3}} \sin \alpha_{3}\right)=0 \\
\left(m_{2} R_{2} \cos \alpha_{2}+\frac{m_{3} R_{3} L_{2}}{L_{3}} \cos \alpha_{3}\right)=0
\end{gathered}
$$

$$
\begin{array}{r}
\left(m_{2} R_{2} \sin \alpha_{2}+\frac{m_{3} R_{3} L_{2}}{L_{3}} \sin \alpha_{3}\right)=0 \\
\left(m_{4} R_{4} \cos \alpha_{4}-\frac{m_{3} R_{3} L_{4}}{L_{3}} \cos \alpha_{3}+m_{3} L_{4}\right)=0 \\
\left(m_{4} R_{4} \sin \alpha_{4}-\frac{m_{3} R_{3} L_{4}}{L_{3}} \sin \alpha_{3}\right)=0
\end{array}
$$

From (12) and (13),

$$
\alpha_{2}=\alpha_{3} \text { and } R_{3}=-\left(\frac{m_{2}}{m_{3}}\right)\left(\frac{L_{3}}{L_{2}}\right) R_{2}
$$

This is an important observation for the mass distribution of links 2 and 3, which have to obey (16) for shaking force balancing. Depending on the mass and the link length ratios, $R_{3}$ is evaluated in terms of negative $R_{2}$, which implies that the locations of the mass centers for link 2 and link 3 are separated from each other by $180^{\circ}$. This might restrict the usable workspace of the manipulator. However, this can be avoided by imposing tight constraints on the sizes of the radial distances $R_{2}$ and $R_{3}$. The remaining four conditions (Eqs.10,11,14,15) are imposed as the constraints which must be satisfied by the balancing parameters while minimizing an optimisation function described in the next subsection. It must be noted these are the same as the conditions for static balancing [17].

\section{B. Shaking Moment Balancing Requirements}

The shaking moment of a system is the time rate of change of the total angular momentum of the system about a pivot point in the mechanism frame. For the mechanism considered in this study, the pivotal point of the first joint is taken as the reference point for the angular momentum. The angular momentum $H_{A}$ of a system consisting of ' $\mathrm{n}$ ' interconnected rigid bodies is;

$$
\vec{H}_{A}=\sum_{i=1}^{n-1} \int\left[\vec{r}_{i} \times\left(\overrightarrow{\dot{\theta}}_{i} \times \vec{r}_{i}\right)\right] d m_{i}+\sum_{i=1}^{n-1} \vec{r}_{i} \times m_{i} \overrightarrow{\dot{r}}_{i}
$$

The angular momentum of the planar mechanism considered in this study is perpendicular to the plane of the movement and is given by;

$$
H_{A}=\sum_{i=1}^{n-1}\left(I_{G}\right)_{i} \dot{\theta}_{i}+\sum_{i=1}^{n-1} m_{i}\left(r_{x} \dot{r}_{y}-r_{y} \dot{r}_{x}\right)_{i}
$$

where $\left(I_{G}\right)_{i}$ is the mass moment of inertia of each moving link about an axis perpendicular to its mass center. The shaking moment transmitted to the frame is

$$
M_{s}=-\frac{d H_{A}}{d t}
$$


From (18), the angular momentum is obtained as

$$
\begin{aligned}
& H_{A}=\dot{\theta}_{1}\left[\left(I_{G}\right)_{1}+m_{1} R_{1}^{2}+m_{2} L_{1}^{2}+m_{2} R_{2} L_{1} \cos \left(\theta_{1}-\theta_{2}-\alpha_{2}\right)\right] \\
& +\dot{\theta}_{2}\left[\left(I_{G}\right)_{2}+m_{2} R_{2}^{2}+m_{2} R_{2} L_{1} \cos \left(\theta_{1}-\theta_{2}-\alpha_{2}\right)\right] \\
& +\dot{\theta}_{3}\left[\begin{array}{l}
\left(I_{G}\right)_{3}+m_{3} R_{3}^{2}+m_{3} R_{3} L_{4} \cos \left(\theta_{4}-\theta_{3}-\alpha_{3}\right) \\
+m_{3} R_{3} L_{0} \cos \left(\alpha_{0}-\theta_{3}-\alpha_{3}\right)
\end{array}\right] \\
& +\dot{\theta}_{4}\left[\begin{array}{l}
\left(I_{G}\right)_{4}+m_{4} R_{4}^{2}+m_{3} L_{4}^{2}+m_{3} R_{3} L_{4} \cos \left(\theta_{4}-\theta_{3}-\alpha_{3}\right) \\
+m_{4} R_{4} L_{0} \cos \left(\alpha_{0}-\theta_{4}-\alpha_{4}\right)+m_{3} L_{4} L_{0} \cos \left(\theta_{4}-\alpha_{0}\right)
\end{array}\right]
\end{aligned}
$$

If the mechanism is driven with a constant angular velocity, the angular momentum of the system can be constant (consequently the shaking moment is zero), provided that the 'cosine' terms in Eq.20 have either a zero or a constant numerical value. In practice, it is almost impossible to satisfy these conditions simultaneously without adding auxiliary linkages to the original mechanism [20-22]. Alternatively, a partial shaking moment balancing can be achieved by minimising the amplitudes of the 'cosine' terms. With this in mind, an optimisation procedure can be employed to minimize the deviation of the angular momentum from its average value throughout the mechanism range of motion.

\section{Objective Function}

As a force balanced mechanism increases the magnitude and variation of the bearing forces and most importantly shaking moment and the driving torques, the following objective function is adopted here

$$
O F=\operatorname{Min}\left\{\begin{array}{l}
w_{1} \sqrt{\sum_{i=1}^{m}\left(F_{B}^{2}+F_{C}^{2}+F_{D}^{2}\right)}+w_{2} \sqrt{\sum_{i=1}^{m}\left(F_{A}^{2}+F_{E}^{2}\right)} \\
+w_{3} \sqrt{\sum_{i=1}^{m}\left(\tau_{1}^{2}+\tau_{4}^{2}\right)}+w_{4} \sqrt{\sum_{i=1}^{m} M_{S}^{2}} \\
+w_{s} \sqrt{\sum_{i=1}^{m}\left(H_{A}-\bar{H}_{A}\right)^{2}}
\end{array}\right\}
$$

where ' $\mathrm{m}$ ' is the number of the discrete values of the manipulator movement, $w_{i}$ are the weighting factors, and $\bar{H}_{A}$ is the average angular momentum. The goal of the optimisation is to determine the numerical values of the balancing parameters $m_{i}, R_{i}, \alpha_{i},\left(I_{G}\right)_{i}$ of the manipulator by minimizing the objective function and satisfying the constraints given in the next subsection.

\section{Constraints}

In order to limit the solution, the objective function is subjected to the following constraints, in addition to the constraints imposed by Eqs. 10,11,14,15:

$$
\begin{aligned}
& \text { 1. }-L_{z} \leq R_{z} \leq L_{z} \text { for } z=1 \cdots 4 \text {, } \\
& \text { 2. } 1 \leq m_{z} \leq 5 \text { for } z=1 \cdots 4,
\end{aligned}
$$

$$
\begin{aligned}
& \text { 3. } 0.01 \leq\left(I_{G}\right)_{z} \leq 0.02 \text { for } z=1 \cdots 4 \text {, } \\
& \text { 4. } 0 \leq \alpha_{z} \leq 180^{\circ} \text { for } z=1 \cdots 4,
\end{aligned}
$$

Hence, dynamic balancing of the manipulator is formulated as a constrained nonlinear optimisation problem. A computer program based on a sequential quadratic programming method is prepared to accomplish the constrained minimization of the $\mathrm{OF}$ as a function of the balancing parameters, starting with an initial value for each parameter.

\section{NUMERICAL RESUI.TS AND DISCUSSION}

It is well known that a mechanism with a poor geometry and transmission angles, which is the angle $\mu$ between $L_{2}$ and $L_{3}$ of Fig. 1, will be likely to have an unsatisfactory performance. With this in mind, the link lengths of the mechanism are obtained from another optimisation procedure based on the minimization of the overall deviation of the condition number of the manipulator Jacobian matrix from the ideal/isotropic condition number throughout the workspace of the manipulator [6], provided that the transmission angle $\mu$ is within the desirable range of $50^{\circ} \leq \mu \leq 130^{\circ}$. The planar parallel manipulator with $\alpha_{0}=0^{\circ}$ and the link lengths of $L_{0}=34.9898, L_{1}=9.7255$, and $L_{2}=24.3222$ has resulted in a transmission angle varying continuously between $62.2630^{\circ}$ and $106.3542^{\circ}$. These link lengths are utilised in this study while determining the optimum values of balancing parameters. With reference to (16), $m_{3}=m_{2}$ is selected such that $R_{3}=-R_{2}$ for the optimum dynamic balancing. Further, it is assumed that $\left(I_{G}\right)_{3}=\left(I_{G}\right)_{2}$.

It is also assumed that the manipulator follows a cycloidal motion profile while it operates between $30^{\circ} \leq \theta_{1} \leq 390^{\circ}$, $\theta_{4}=0.8 \theta_{1}$, with step sizes of $5^{\circ}$ (i.e., $\mathbf{n}=72$ ). A cycloidal motion profile, which has smooth first and second order derivatives, and a finite third derivative, is expressed by

$$
\theta_{1}(k)=30^{\circ}+360^{\circ}\left[\frac{k}{n}-\frac{1}{2 \pi} \sin \left(2 \pi \frac{k}{n}\right)\right], \quad k=0 \cdots n
$$

Such a motion profile does not impart any sudden motion to the manipulator. We use a performance criterion to evaluate the efficiency of the balancing procedure, which we call SumSquared Value (SSV):

$$
S S V=\sqrt{\sum_{k=0}^{n} G(k)^{2}}
$$

where $G$ indicates each of the dynamic quantities (i.e., the bearing forces, the driving torques, the shaking moment, the angular momentum, and the shaking force) involved in the balancing procedure. Depending on the value of the weighting factors, different combinations of the five components of the 
TABLE I

THE SOLUTIONS OBTAINED FROM THE CONSTRAINED OPTIMUM BALANCING FOR THE INITIAL CONDITIONS OF $L_{1} / 2, L_{2} / 2, L_{4} / 2,2.0,1.0,2.0,0.002,0.02,0.002 \pi / 10, \pi / 10, \pi / 10$.

\begin{tabular}{|c|c|c|}
\hline $\begin{array}{c}\text { Weighting Factors } \\
{\left[w_{1}, w_{2}, w_{3}, w_{4}, w_{5}\right]}\end{array}$ & {$\left[R_{1}, R_{2}, R_{4}, m_{1}, m_{2}, m_{4},\left(I_{G}\right)_{1},\left(I_{G}\right)_{2},\left(I_{G}\right)_{4}, \alpha_{1}, \alpha_{2}, \alpha_{4}\right]$} & $\begin{array}{c}\text { Sum-Squared Values (SSV) } \\
{\left[\begin{array}{l}F_{A}, F_{B}, F_{C}, F_{D}, F_{E}, \tau_{1}, \\
\tau_{A}, M_{S}, H_{A}, F_{S}\end{array}\right] \times 10^{3}}\end{array}$ \\
\hline $\begin{array}{c}\text { (a) } \\
{[1,1,1,1,1]}\end{array}$ & $-9.7255,-0.0846,9.7253,1,1,1,0.001,2,0.001,0.0035,1.5641,3.1381$ & $\begin{array}{cccccc}0.1676 & 0.0838 & 0.0003 & 0.0838 & 0.1676 \\
0.0193 & 0.0189 & 0.0173 & 0.2451 & 0.0\end{array}$ \\
\hline$[0,1,1,1,1]$ & $-9.7255,-0.0026,9.7254,1,1,1,0.001,1.1979,0.001,0.0001,1.5048,3.1415$ & $\begin{array}{cccccc}0.1676 & 0.0838 & 0.0001 & 0.0838 & 0.1676 \\
0.0192 & 0.0190 & 0.0173 & 0.2452 & 0.0 \\
\end{array}$ \\
\hline $\begin{array}{c}(c) \\
{[0,0,1,1,1]}\end{array}$ & $-1.9451,-0.0004,1.9451,5,1,5,0.001,0.01,0.001,0.0,1.2216,3.1416$ & $\begin{array}{cccccc}0.5029 & 0.0838 & 0.0000 & 0.0838 & 0.5029 \\
0.0115 & 0.0114 & 0.0104 & 0.1157 & 0.0 \\
\end{array}$ \\
\hline $\begin{array}{c}\text { (d) } \\
{[0,0,0,1,1]}\end{array}$ & $-2.0616,-1.4562,-1.8286,5,1,5,0.001,0.01,0.001,0.0,0.0,0.0$ & $\begin{array}{cccccc}0.5801 & 0.3707 & 0.3736 & 0.3948 & 0.6697 \\
2.5222 & 2.8993 & 0.0105 & 0.1156 & 0.0 \\
\end{array}$ \\
\hline $\begin{array}{c}(\mathrm{e}) \\
{[1,1,0,0,0]}\end{array}$ & $-8.4096,-0.5224,-8.4175,1.184,1.0023,1.1332,0.002,0.3046,0.002,6.2832,0,0$ & $\begin{array}{ccccc}0.2105 & 0.1476 & 0.1344 & 0.1686 & 0.2393 \\
0.9107 & 1.0391 & 0.0162 & 0.2233 & 0.0 \\
\end{array}$ \\
\hline$[0,0,1,0,0]$ & $-1.9451,-0.0005,8.0002,5,1,1.2156,0.001,0.01,0.01,0.0,0.2358,3.1416$ & $\begin{array}{cccccc}0.5029 & 0.0838 & 0.0001 & 0.0838 & 0.1857 \\
0.0117 & 0.0172 & 0.0131 & 0.1646 & 0.0 \\
\end{array}$ \\
\hline $\begin{array}{c}(\mathrm{g}) \\
{[0,0,0,1,0]}\end{array}$ & $-1.9744,-0.4317,-1.916,5,1,5,0.001,2,0.001,6.2738,5.7188,0.0096$ & $\begin{array}{ccccc}0.4975 & 0.1162 & 0.0938 & 0.1347 & 0.5252 \\
0.6365 & 0.7269 & 0.0103 & 0.1168 & 0.0 \\
\end{array}$ \\
\hline$[0,1,0,0,0]$ & $-9.7255,0.0,-9.7255,1,1,1,0.002,0.9018,0.002,6.2832,1.8634,0.0$ & $\begin{array}{ccccc}0.1676 & 0.0838 & 0.0000 & 0.0838 & 0.1676 \\
0.0191 & 0.0191 & 0.0173 & 0.2452 & 0.0 \\
\end{array}$ \\
\hline $\begin{array}{c}\text { (i) } \\
{[0,1,0,1,0]}\end{array}$ & $9.7255,0.3791,-9.7255,1.0001,1,1.0002,0.001,2,0.001,3.126,1.5667,0.0156$ & $\begin{array}{ccccc}0.1675 & 0.0837 & 0.0012 & 0.0840 & 0.1678 \\
0.0187 & 0.0197 & 0.0172 & 0.2458 & 0.0 \\
\end{array}$ \\
\hline$[0,1,1,1,0]$ & $-9.7255,0.0005,9.7255,1,1,1,0.001,2,0.001,0.0,1.4232,3.1416$ & $\begin{array}{llllll}0.1676 & 0.0838 & 0.0000 & 0.0838 & 0.1676 \\
0.0190 & 0.0191 & 0.0173 & 0.2452 & 0.0 \\
\end{array}$ \\
\hline $\begin{array}{c}(\mathrm{k}) \\
{[0,1,1,0,1]}\end{array}$ & $-9.7255,-0.0005,9.7254,1,1,1,0.001,0.01,0.001,0.0,1.2137,3.1416$ & $\begin{array}{ccccc}0.1676 & 0.0838 & 0.0000 & 0.0838 & 0.1676 \\
0.0192 & 0.0190 & 0.0173 & 0.2452 & 0.0 \\
\end{array}$ \\
\hline$[0,0,1,1,0]$ & $-3.3933,-0.0001,4.1134,2.8661,1,2.3644,0.01,2,0.001,0.0,1.5466,3.1416$ & $\begin{array}{cccccc}0.3240 & 0.0838 & 0.0000 & 0.0838 & 0.2820 \\
0.0129 & 0.0136 & 0.0120 & 0.1446 & 0.0 \\
\end{array}$ \\
\hline
\end{tabular}

objective function described by (21) are used to determine the balancing parameters. Twelve sets of optimisation results are obtained for the same initial conditions and these are given in Table I. Note that the units are arbitrary length, mass, and mass moment of inertia units. But the units for the angles are radian. These values describe the numerical values of the balancing parameters $\left(m_{z}, R_{z},\left(I_{G}\right)_{z}, \alpha_{z}\right)$. As typical representatives of optimisation results, the variation of the reaction/bearing forces with $\theta_{1}$ is depicted in Fig. 2 for the solutions given in the second (Case a), fourth (Case c), and sixth (Case e) rows of the Table I. The corresponding driving torques, shaking moment, angular momentum, shaking force, and linear momentum variations are provided in Fig. 3.

When the shaking moment and the driving torque components of the $\mathrm{OF}$ are not considered during the optimisation procedure (Case e), not only the bearing forces, but also the driving torques increase significantly, as seen in the plots indicated with ' $e$ ' in Figs. 2 and 3. This follows that a force-balanced mechanism does not necessarily require less powerful and smaller actuators to move the mechanism $[11,17]$. When the shaking moment and the driving torque components of the OF are considered together with the ground reaction forces (Case $\mathrm{j}$ ), it is found that the resulting SSVs are comparable to those of 'Case $a$ '. When the shaking moment component only (Case $\mathrm{g}$ ) is considered, both bearing forces and the driving torques increase dramatically; comparing their SSVs with those of the 'Case $j$ '. When the variation of the angular momentum from its average value is considered in place of the shaking moment balancing (Case k), the resulting SSVs become the same as those of 'Case $j$ '. When only the shaking moment and driving torque minimization are considered (Case 1), the bearing forces, especially the ground reaction forces can increase and can trigger the vibration of the mechanism frame; comparing the SSVs of these forces to those of 'Case $j$ ' and 'Case a'. The same finding can be seen in 'Case c'.
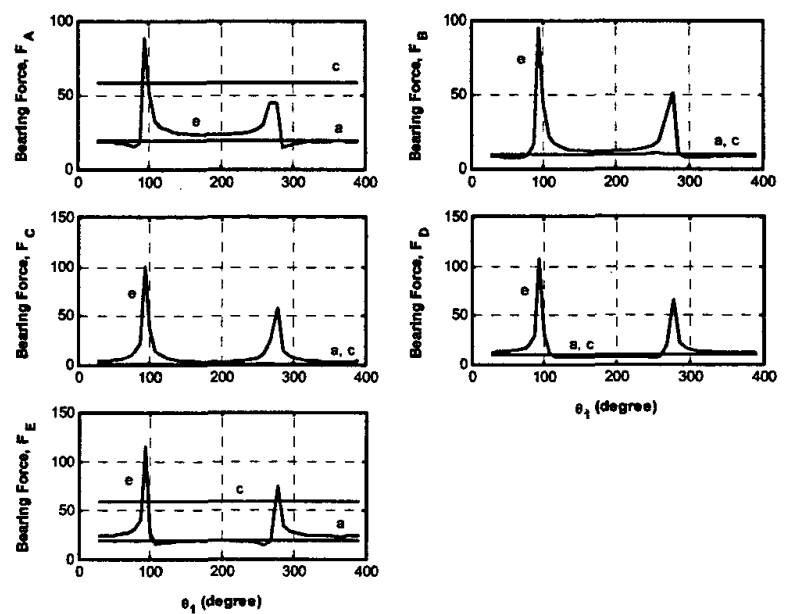

Figure 2. The variation of reaction forces with $\theta_{1}$ for the optimum balancing parameters given in Table $I$. 

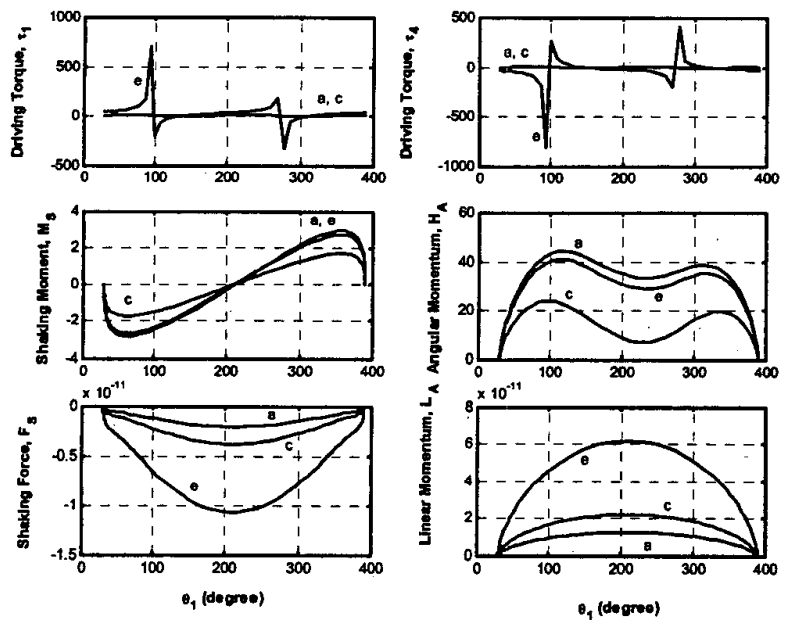

Figure 3. The variation of the driving torques, shaking moment, angular momentum, shaking force, and linear momentum with $\theta_{1}$ for the optimum balancing parameters given in Table $\mathrm{I}$.

\section{CONCLUSIONS}

We have presented an optimum dynamic balancing method based on the minimization of the sum squared values of the bearing forces, driving torques, shaking moment, and the deviation of the angular momentum from its mean value throughout a practical operation range of the manipulator, provided that a set of balancing constraints consisting of shaking force balancing conditions, the sizes of inertial and geometric parameters are satisfied. Sets of optimisation results corresponding to various combinations of the elements of the objective function are accomplished in order to quantify their influence on the resulting bearing forces, the driving torques, the shaking moment and force associated with the parallel manipulator. The results prove that the proposed optimisation approach can be used to minimize any desired combination of the forces, moments, and torques involved in the parallel mechanism by choosing a suitable set of weighting factors. The method is systematic, versatile and easy to implement for the optimum dynamic balancing of parallel manipulators.

\section{REFERENCES}

[1] K. H. Hunt, "Structural kinematics of in parallel-actuated robot-arms", Transactions of ASME, J. of Mechanisms, Transmissions, and Automation in Design, Vol:105, pp. $705-712$, December 1983.

[2] D. C. Tao and A.S. Tall, "Analysis of a symmetrical five-bar linkage", Product Engineering, Vol:23, pp.175-177, 201,203,205, January 1952.
[3] S. E. Rose, "Five-bar loop synthesis", Machine Design, Vol:33, pp. 189 195, October 12, 1961.

[4] E. P. Pollitt, "Five-bar linkages witl two drive cranks", Machine Design, Vol:34, pp. $168-179$, January $18,1962$.

[5] T. W. Lee and F. Freudenstein, "Design of Geared 5-Bar Mechanisms for Unlimited Crank Rotations and Optimum Transmission", Mechanism and Machine Theory, Vol:13, pp. 235 - 244, 1978.

[6] G. Alici and B. Shirinzadeh, "Constrained structural optimisation of a revolute-jointed planar parallel manipulator", 2003 IEEE/ASME International Conference on Advanced Intelligent Mechatronics, pp. 1244-1249, July 2003, Japan.

[7] M. Skreiner, "Dynamic analysis used to complete the design of a mechanism", J. of Mechanisms, Vol. 5, pp.105-119, 1970.

[8] G. G. Lowen, F. R. Tepper, and R. S. Berkof, "Balancing of linkages - An update", Mech. and Machine Theory, Vol:18, No. 3, pp. 213-220, 1983.

[9] R. S. Berkof, and G. G. Lowen, "A new method for completely force balancing simple linkages", ASME J. of Engineering for Industry, Vol.91, pp. $21-26$, February 1969.

[10] C. Bagci, "Complete balancing of space mechanisms - shaking force balancing", ASME J. of Mechanisms, Trans., and Auto. in Design, Vol.110, No.12, pp. 609-616, December 1983.

[11] T. Laliberte, C. M. Gosselin, and M. Jean, "static balancing of 3-DOF planar parallel mechanisms", IEEE ' ASME Trans. on Mechatronics, Vol: 4, No: 4, pp. 363 - 377, December 1999.

[12] B. Porter, and D. J. Sanger, "Synthesis of dynamically optimum four-bar linkages", Procs. of Conference on Mechanisms, Paper C69/72, pp. 24 28, Institution of Mechanical Engineers, 1972.

[13] T. W. Lee, and C. Cheng, "Optimum balancing of combined shaking force, shaking moment, and torque fluctuations in high-speed linkages", ASME J. of Mechanisms, Trans., and Auto. in Design,Vol. 106, pp. 242 - 251, June 1984.

[14] H. C. Yen, "Balancing of high speed rnachinery", ASME J. of Engineering for Industry, Vol.89, pp. $111-118$, Februiry 1967.

[15] G. G. Lowen, and R. S. Berkof, "Survey of investigations into the balancing of linkages", $J$. of Mechanisms, Vol.3, No.4, pp.221 - 231, 1968.

[16] G. Alici, "An Inverse Position Analysis of Five-bar Planar Parallel Manipulators", Robotica, 20:2, pp.195--201, 2002.

[17] G. Alici, and B. Shirinzadeh, "Optimum force balancing with mass distribution and a single elastic element for a five-bar parallel manipulator", IEEE International Conference on Robotics and Automation, pp. 3666 - 3671, Taipei, Taiwan, September 2003.

[18] J. L. Wiederrich, and B. Roth, "Momentum balancing of linkages", Transactions of the ASME Journal of Engineering for Industry, Vol.96, pp. 1289 - 1295, November 1976.

[19] F. L. Conte, G. R George, R. W. Mayne, and J. P. Sadler, "Optimum mechanism design combining kinematic and dynamic force considerations", Transactions of the ASME Journal of Engineering for Industry, Vol.95, pp. 662 - 670, May 1975.

[20] G. Feng, "Complete shaking force and shaking moment balancing of 26 types of four-, five-, and six-bar linkages with prismatic pairs", Mech. and Machine Theory, Vol:25, No. 2, pp. 183 - 192, 1990.

[21] V. Arakelian, and M. Dahan, "Partial shaking moment balancing of fully force balanced linkages", Mech. and Machine Theory, Vol:36, pp. 1241 $1252,2001$.

[22] S. K. Agrawal, and A. Fattah, "Reactionless space and ground robots: novel designs and concept studies", Mech. and Machine Theory, Vol:39, pp. $25-40$, January 2004.

[23] J. L. Elliott, and D. Tesar, "The theory of torque, shaking force, and shaking moment balancing of four link mechanisms", Transactions of the ASME Journal of Engineering for Industry, Vol.97, pp. 715 - 722, August 1977. 